\title{
Dieta de roedores sigmodontinos (Cricetidae) en los bosques montanos tropicales de Huánuco, Perú
}

\author{
Diet of Sigmodontine rodents (Cricetidae) in tropical montane forests \\ from Huánuco, Peru
}

\author{
Maggie C. Noblecilla ${ }^{1}$ y Víctor Pacheco ${ }^{1,2}$
}

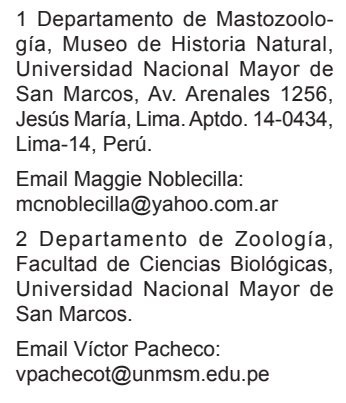

tozoología, Museo de Historia Natural, San Marcos, Av. Arenales 1256, Jesús María, Lima. Aptdo. 14-0434,

Email Maggio Noblecilla: mcnoblecilla@yahoo.com.a Departamento de Zoología, Universidad Nacional Mayor de San Marcos.

vpachecot@unmsm.edu.pe

Presentado: $\quad$ 07/09/2012 Aceptado: $\quad$ 25/11/2012 Publicado online: $15 / 01 / 2013$

\begin{abstract}
Resumen
Se analizó el contenido estomacal de cinco especies de roedores sigmodontinos: Akodon orophilus, Microryzomys altissimus, M. minutus, Thomasomys notatus y T. kalinowskii, procedentes de los bosques montanos de Huánuco, Perú (2564 - $3850 \mathrm{~m}$ de altitud). Concluimos que $A$. orophilus es insectívora por haber presentado un alto volumen de artrópodos (adultos y larvas) en el contenido estomacal $(90,1 \%)$; mientras que $T$. notatus y T. kalinowskii son principalmente herbívoras por el alto volumen de materia vegetal, $89 \%$ y $67,75 \%$ respectivamente; y que $M$. altissimus y $M$. minutus son omnívoras por presentar volúmenes similares tanto para vegetales como para artrópodos. Thomasomys kalinowskii es considerada generalista por la mayor amplitud de nicho $(4,61)$, mientras $A$. orophilus es considerada especialista por el menor valor $(1,70)$. Akodon orophilus mostró una preferencia por el consumo de artrópodos adultos al tener un bajo coeficiente de variación $(C V=20 \%)$ y también un significativo aumento en el consumo de larvas de artrópodos en la época húmeda, siendo la única especie con variación estacional en la dieta. Por otro lado, la sobreposición de nicho fue menor a 0,75 en el $80 \%$ de los pares de especies comparados, indicando una baja similitud en la dieta. La mayor similitud en la dieta se observó entre $M$. altissimus - T. notatus $(0,822)$ y M. minutus - T. kalinowskii $(0,816)$. Se concluye que estos roedores sigmodontinos, simpátricos en los bosques montanos de Huánuco, exhiben dietas disímiles, probablemente como una estrategia para evitar o aminorar la competencia interespecífica.
\end{abstract}

Palabras clave: Dieta de roedores; Akodon; Microryzomys; Thomasomys; Sigmodontinae; bosque montano; Huánuco; Perú.

\section{Abstract}

We analyzed the stomach contents of five species of sigmodontine rodents: Akodon orophilus, Microryzomys altissimus, M. minutus, Thomasomys notatus, and T. kalinowskii, from mountain forests of Huánuco, Perú (2564 $-3850 \mathrm{~m}$ ). We found that $A$. orophilus is an insectivorous species because the high volume of arthropods (adults and larvae) in the stomach contents (90.1\%); T. notatus and T. kalinowskii are primarily herbivorous because they had a high volume of plant material of $89 \%$ and $67.75 \%$ respectively; whereas $M$. altissimus and M. minutus are omnivorous because they presented similar volume percentages for plants and arthropods. T. kalinowskii is considered a generalist species because it had the highest niche breadth (4.61), whereas $A$. orophilus is considered a specialist because it had the lowest value (1.70). Akodon orophilus registered a low coefficient of variation ( $C V=20 \%$ ) showing a preference for consuming adult arthropod, and also a significatively high consumption of arthropod larvae in the wet season, being the only species with a seasonal variation in the diet. On the other hand, the niche overlap was less than 0.75 in $80 \%$ of species pairs indicating low similarity in the diet, but greater than 0.75 between $M$. altissimus - T. notatus $(0.822)$ and $M$. minutus - T. kalinowskii $(0.816)$ suggesting a higher diet similarity. We conclude that these sigmodontine rodents, sympatric in the montane forests of Huánuco, exhibit dissimilar diets, probably as a strategy to prevent or lessen interspecific competition.

Keywords: Rodent diet; Akodon; Microryzomys; Thomasomys; Sigmodontinae; montane forest; Huánuco; Peru.

\section{Introducción}

Perú posee una gran diversidad de roedores, conociéndose no menos de 162 especies (Pacheco et al. 2009); sin embargo, pocas especies cuentan con estudios de contenido y variabilidad de su dieta. Después de los estudios pioneros de Dorst (1972) y Pizzimenti y De Salle (1980) muy pocos trabajos sobre análisis de dieta han sido publicados, como los de Guabloche et al. (2002) con Aegialomys xanthaeolus (reportado como Oryzomys xantheolus) y Solari (2007) con roedores altoandinos del Parque Nacional del Manu. Otros reportes cualitativos de dieta en algunas especies incluyen a Luna y Patterson (2003) para Rhagomys longilingua y Pacheco y Peralta (2011) para Rhipidomys ochrogaster. Regionalmente, la mayoría de estos estudios fueron realizados en ambientes de puna o desiertos, exceptuando los dos últimos trabajos que involucraron a especies de bosques montanos.
Los bosques montanos en el Perú albergan un alto número de endemismos y es el segundo ambiente con mayor diversidad de mamíferos (Pacheco 2002, Pacheco et al. 2009). Aunque existen varios estudios de la diversidad de roedores en esta ecorregión (e.g., Amanzo et al. 2003, Pacheco et al. 2008, Pacheco et al. 2011), el conocimiento de la dieta en estas especies es prácticamente nulo, excepto por los estudios de Luna y Patterson (2003) en Rhagomys longilingua y Solari (2007) en Akodon torques, ambas determinadas como especies insectívoras; y Pacheco y Peralta (2011) con Rhipidomys ochrogaster que fue determinada como omnívora. Para otras especies y géneros se debe recurrir a extrapolaciones en base a estudios realizados en países cercanos o vecinos como $\mathrm{Ar}$ gentina y Chile (e.g., Brandán 1995, Giannoni et al. 2005, López-Cortés et al. 2007). 
En este trabajo presentamos el primer estudio sobre la dieta de un ensamblaje de roedores sigmodontinos en los bosques montanos centrales de Perú y que toma en consideración el sexo y la época de colecta; examinándose además el grado de competencia y similitud entre ellos.

\section{Material y métodos}

Se analizó la dieta de 105 roedores sigmodontinos, de un total de 325, en base al análisis del contenido estomacal de estómagos llenos (Pizzimenti \& De Salle 1980): Akodon orophilus (37), Microryzomys altissimus (5), M. minutus (9), Thomasomys notatus (6) y T. kalinowskii (48); provenientes de los bosques montanos de la Región Huánuco, Perú (localidades Ucumaria, Shogos, Chaupiloma, Galloganá, Chinchuragra, Iscarag, Kenwarajra, Hatuncucho, Pampa Hermosa y los bosques colindantes a los campamentos Regional y Provias; entre los 2890 y 3850 m). Estos especímenes fueron colectados mediante un muestreo estándar en el periodo de septiembre-octubre de 2005 y marzo-junio de 2006 en un área correspondiente al ecosistema de Bosque Montano (sensu Young y Valencia 1992), con las siguientes unidades de vegetación: Vega de ciperáceas, Pajonal, Bosque montano primario (que comprende bosques densos) y Bosque montano disturbado (que comprende sembríos entre bosques poco densos) (Pacheco et al. 2006).

Análisis de la dieta.- Los estómagos fueron separados del tracto digestivo, su contenido homogenizado (Meserve 1981) y luego separado en dos fracciones: partículas pequeñas $(<0,7$ $\mathrm{mm}$ ) y partículas grandes (>0,7 $\mathrm{mm}$ ) (Guabloche et al. 2002). Cada fracción fue examinada usando un estéreomicroscopio a 40 aumentos en no menos de cinco campos ópticos utilizándose el porcentaje de cobertura de campo como una medida del volumen (Solari 1997).

Se empleó seis categorías alimenticias: larvas de artrópodos, artrópodos adultos, monocotiledóneas, dicotiledóneas, tejido vascular y la categoría otros (v.g., hongos, pelo, cebo, tierra). Se obtuvieron porcentajes promedios de cada categoría para cada individuo, los cuales a su vez se agruparon para obtener promedios generales de cada categoría para cada especie.

Para evidenciar el grado de especialización en la dieta se utilizó el coeficiente de variación (CV) para cada categoría alimenticia en cada especie con 9 o más ejemplares; considerándose un valor de CV menor a 25\% como indicativo de alguna preferencia para las categorías alimenticias (Solari 2007). Se analizó la variación en la dieta según el sexo en Akodon orophilus y Thomasomys kalinowskii y según la época de colecta en $A$. orophilus, especies que tuvieron la mayor cantidad de muestra. Se estimó también el nivel de variación de uso del recurso, en la dieta de cada especie, a fin de señalar la existencia de patrones especialistas o generalistas, mediante la medida de amplitud de nicho estimada con el Índice de Levins (Levins 1968):

$$
B=\frac{1}{\sum p_{j}^{2}}
$$

Donde: $p_{j}$ representa la proporción del recurso $j$ en la dieta. El índice de Levins varía entre un mínimo de 1 y un máximo igual al número $(\mathrm{N})$ de categorías alimenticias (en este caso 6).

Utilizando el porcentaje de volumen de cada categoría alimenticia se obtuvo el Índice de Sobreposición Simétrico de Pianka $\left(O_{j k}\right)$ (Pianka 1973) y el Índice Modificado de Morisita $\left(C_{H}\right)$ (Horn 1966), para indicar el grado de competencia por el uso de recursos alimenticios específicos (Sale 1974):

$$
\begin{aligned}
O_{j k} & =\frac{\sum p_{j} p_{k}}{\sqrt{\sum p_{j}^{2} \sum p_{k}^{2}}} \\
C_{H} & =\frac{2 \sum p_{j} p_{k}}{\sum p_{j}^{2}+\sum p_{k}^{2}}
\end{aligned}
$$

Donde: $p_{i j}$ y $p_{i k}$ representan la proporción de la categoría alimenticia $i$ en la dieta de las especies $j$ ó $k$. El Índice de Sobreposición se consideró alto para valores sobre 0,75 y bajo para aquellos menores de 0,50 (Solari 2007). El análisis estadístico incluye a las especies con un número de ejemplares mayor a 10. Se aplicó la prueba U de Mann-Whitney para determinar, en cada especie, si existen diferencias significativas en el consumo de cada una de las seis categorías alimenticias con relación al sexo y la época de colecta, con el programa IBM SPSS Statistics v.20.

\section{Resultados}

Nuestros datos, basados en el análisis del contenido de 105 estómagos, muestran una alta proporción de consumo de artrópodos (adultos y larvas) en Akodon orophilus (90,1\%), un alto porcentaje de material vegetal en Thomasomys notatus $(89,2 \%)$, un porcentaje relativamente alto de materia vegetal (principalmente semillas) en T. kalinowskii $(67,7 \%)$ y una aparente tendencia hacia la omnivoría en Microryzomys altissimus y $M$. minutus, con porcentajes equivalentes para vegetales y artrópodos (Tabla 1).

Tabla 1. Hábitos alimenticios para 5 especies de roedores de los Bosques Montanos de Huánuco. El porcentaje de consumo de cada categoría

\begin{tabular}{|c|c|c|c|c|c|}
\hline Categoría alimenticia & A. orophilus $(\mathrm{N}=37)$ & M. altissimus $(\mathrm{N}=5)$ & M. minutus $(\mathrm{N}=9)$ & T. kalinowskii $(\mathrm{N}=48)$ & T. notatus $(\mathrm{N}=6)$ \\
\hline Artrópodos Larva & $15,35(13,61) 0,89$ & $15,13(10,94)-$ & $2,96(3,67) 1,24$ & $8,90(6,39) 0,72$ & $2,24(1,17)-$ \\
\hline Artrópodo Adulto & $74,74(14,91) 0,20$ & $26,58(19,58)-$ & $35,92(25,44) 0,71$ & $12,63(7,34) 0,58$ & $1,50(2,32)-$ \\
\hline Monocotiledóneas & $1,75(3,46) 1,98$ & $42,97(28,78)-$ & $16,20(21,29) 1,31$ & $16,04(17,63) 1,10$ & $60,13(46,58)-$ \\
\hline Dicotiledóneas & $0,32(1,21) 3,82$ & $5,97(8,93)-$ & $25,05(26,36) 1,05$ & $36,43(22,12) 0,61$ & $21,79(35,36)-$ \\
\hline Tejido vascular & $0,35(1,12) 3,19$ & $2,26(3,61)-$ & $4,11(9,06) 2,21$ & $15,27(9,45) 0,62$ & $7,29(17,85)-$ \\
\hline Otros & $7,50(3,57) 0,48$ & $7,10(0,90)-$ & $15,75(11,31) 0,72$ & $10,72(5,51) 0,51$ & $7,05(1,94)-$ \\
\hline Amplitud de nicho & 1,70 & 3,48 & 4,08 & 4,61 & 2,38 \\
\hline
\end{tabular}
alimenticia es expresado como promedio, con la desviación estándar en paréntesis y el coeficiente de variación (para especies con 9 o más individuos). Se incluye además la Amplitud de Nicho basado en el Índice de Levins para cada especie. 
Tabla 2. Promedio de consumo de cada categoría alimenticia (\%) de Akodon orophilus y Thomasomys kalinowskii, en relación con el sexo ( $\mathrm{m}=$ macho, $\mathrm{h}=$ hembra).

\begin{tabular}{|c|c|c|c|c|}
\hline \multirow{2}{*}{ Categoría alimenticia } & \multicolumn{2}{|c|}{ Akodon orophilus $(\mathrm{N}=37)$} & \multicolumn{2}{|c|}{ Thomasomys kalinowskii $(\mathrm{N}=48)$} \\
\hline & $(m=23)$ & $(\mathrm{h}=14)$ & $(m=28)$ & $(\mathrm{h}=20)$ \\
\hline Artrópodo larva & 17,47 & 11,86 & 8,57 & 9,36 \\
\hline Artrópodo adulto & 72,36 & 78,65 & 12,23 & 13,20 \\
\hline Monocotiledóneas & 1,85 & 1,58 & 17,62 & 13,84 \\
\hline Dicotiledóneas & 0,35 & 0,27 & 35,46 & 37,79 \\
\hline Tejido vascular & 0,21 & 0,58 & 15,72 & 14,64 \\
\hline Otros & 7,76 & 7,06 & 10,40 & 11,16 \\
\hline
\end{tabular}

Akodon orophilus consumió principalmente artrópodos adultos $(74,7 \%)$, seguido de larvas de artrópodos (15,3\%). En los orizominos $M$. altissimus y $M$. minutus el consumo de material vegetal fue relativamente alto, con un promedio de consumo de $51 \%$ y $45 \%$ respectivamente, prefiriendo además artrópodos adultos sobre las larvas. Thomasomys notatus es considerado una especie herbívora ya que mostró un alto porcentaje de material vegetal (89,2\%), especialmente de monocotiledóneas (60,13\%). De forma similar, la dieta de T. kalinowskii fue principalmente herbívora con $67,7 \%$ del volumen total conformado por material vegetal, aunque no mostró una preferencia por algún ítem en particular (Tabla 1).

La Amplitud de nicho (Índice Levins) varió desde 1,70 en Akodon orophilus hasta 4,61 en Thomasomys kalinowskii. Según estos valores, $A$. orophilus es considerado como especialista y $T$. kalinowskii como generalista. Las demás especies obtuvieron valores intermedios: T. notatus $(2,38)$, Microryzomys altissimus $(3,48)$, M. minutus $(4,08)$ (Tabla 1$)$.

El análisis del coeficiente de variación fue bajo en $A$. orophilus (CV $=19,9 \%)$, sugiriendo la preferencia por artrópodos adultos en su dieta (Tabla 1). En las otras especies y categorías el CV fue generalmente mayor al 50\%.

Se identificó además las partes encontradas en el contenido estomacal al mayor nivel taxonómico posible (Anexo 1). Así, la dieta de $A$. orophilus consistió de poáceas y ciperáceas y una diversidad de insectos que incluye coleópteros, hemípteros, lepidópteros, dípteros, himenópteros, ftirapteros, dipluras y arácnidos; la de $M$. altissimus consistió de semillas de poáceas y carábidos; la de $M$. minutus consistió de poáceas, ciperáceas y coleópteros indeterminados; la de T. notatus consistió de poáceas, ciperáceas y coleópteros carábidos; mientras que en T. kalinowskii se encontró restos de coleópteros y restos vegetales de poáceas, ciperáceas, asteráceas y piperáceas.

Tabla 3. Promedio de consumo de cada categoría alimenticia (\%) de Akodon orophilus en relación con la época ( $\mathrm{s}=$ seca, $\mathrm{h}=$ húmeda). Se observa que existe diferencia estacional significativa en el consumo de Artrópodo larva en Akodon orophilus, usando la prueba $U$ de Mann-Whitney $(p=0,011)$, representado por $\left(^{*}\right)$.

\begin{tabular}{lcc}
\hline \multirow{2}{*}{$\begin{array}{l}\text { Categoría } \\
\text { alimenticia }\end{array}$} & \multicolumn{2}{c}{ Akodon orophilus $(\mathrm{N}=37)$} \\
\cline { 2 - 3 } & $(\mathrm{s}=29)$ & $(\mathrm{h}=8)$ \\
\hline Artrópodo larva & 12,58 & $25,36^{*}$ \\
Artrópodo adulto & 77,27 & 65,58 \\
Monocotiledóneas & 2,02 & 0,78 \\
Dicotiledóneas & 0,32 & 0,31 \\
Tejido vascular & 0,45 & - \\
Otros & 7,37 & 7,96 \\
\hline
\end{tabular}

En cuanto a las cantidades consumidas, A. orophilus consumió principalmente larvas de artrópodos en especímenes machos, mientras que las hembras tuvieron preferencia por los artrópodos adultos. Por otro lado, T. kalinowskii priorizó el consumo de material vegetal sobre los artrópodos, presentando un consumo ligeramente mayor de monocotiledóneas en machos (Tabla 2). Sin embargo, las pruebas U de Mann-Whitney concluyen que no existen diferencias significativas en el consumo de las categorías alimenticias en ambas especies $(\mathrm{p}<0,05)$.

En el análisis de las categorías alimenticias en relación a la época, $A$. orophilus presentó un alto consumo de artrópodos ( $90 \%$ o más) y un bajo porcentaje de material vegetal; y un significativo aumento en el consumo de artrópodos larvas en la época húmeda $(\mathrm{p}<0,05)$ (Tabla 3). En T. kalinowskii no se determinó la relación entre el nivel de consumo de las categorías alimenticias y la época, debido a la ausencia de especímenes en el muestreo de época húmeda.

Uso y sobreposición de recursos alimenticios.- El Índice de sobreposición de Pianka varió desde 0,06 entre $A$. orophilus y $T$. notatus hasta valores altos encima de 0.75 entre $M$. altissimus - T. notatus $(0,822)$ y $M$. minutus - T. kalinowskii $(0,816)$, sugiriendo una alta similaridad en la dieta de las referidas especies (Tabla 4). Estas tendencias son también mostradas en el Índice de Morisita (Tabla 4).

\section{Discusión}

Nuestros resultados indican que los roedores Thomasomys notatus y T. kalinowskii son predominantemente herbívoros, siendo este el primer trabajo donde se reporta el nicho herbívoro en este género y el primero donde un roedor sigmodontino ocupa este nicho en los bosques nublados de los Andes. Anteriormente en Thomasomys sp. de Colombia, Lopez-Arevalo et al. (1993)

Tabla 4. Valores de los Índices de Sobreposición de nicho (Pianka y Morisita) para pares de especies, en base a sus hábitos alimenticios.

\begin{tabular}{lcc}
\hline Pares de especies & $\begin{array}{c}\text { Índice de } \\
\text { Pianka }\end{array}$ & $\begin{array}{c}\text { Índice de } \\
\text { Morisita }\end{array}$ \\
\hline A. orophilus - M. altissimus & 0,572 & 0,537 \\
A. orophilus - M. minutus & 0,759 & 0,693 \\
A. orophilus - T. notatus & 0,063 & 0,062 \\
A. orophilus - T. kalinowskii & 0,088 & 0,3 \\
M. altissimus - M. minutus & 0,741 & 0,738 \\
M. altissimus - T. notatus & 0,822 & 0,807 \\
M. altissimus - T. kalinowskii & 0,596 & 0,59 \\
M. minutus - T. notatus & 0,536 & 0,517 \\
M. minutus - T. kalinowskii & 0,816 & 0,815 \\
T. notatus - T. kalinowskii & 0,657 & 0,623 \\
\hline
\end{tabular}


reportaron una dieta compuesta principalmente de hojas jóvenes y otras partes verdes de plantas, pero el estudio estuvo basado en un solo ejemplar. Estos autores determinaron también la dieta de T. laniger considerándola como frugívora-insectívora. Aunque Thomasomys notatus y T. kalinowskii aparentan compartir el mismo nicho, T. notatus consume en su mayoría monocotiledóneas y T. kalinowskii semillas de dicotiledóneas de la familia Piperaceae, sugiriéndose que ambas especies no compiten por recursos alimenticios. Esta diferencia en dieta podría estar en relación al estrato de vegetación donde generalmente se encuentran ambas especies. Pacheco (en prensa) menciona que $T$. kalinowskii es terrestre y que $T$. notatus podría ser arborícola o semiarborícola. Thomasomys notatus presentó en cambio una alta sobreposición de nicho con $M$. altissimus $(0,82)$, es decir dietas similares y una posible competencia por recursos.

Thomasomys notatus no fue considerada especialista a pesar del alto consumo de monocotiledóneas $(60,1 \%)$ y de tener la amplitud de nicho más baja después de Akodon orophilus $(2,38)$; pero estos datos indican que es la más especialista de las dos especies de Thomasomys. Un análisis con mayor número de muestras es necesario para confirmar nuestros resultados.

Thomasomys kalinowskii es predominantemente herbívora, pero no muestra preferencia entre los distintos ítems vegetales que consume, por ello el Índice de Levins $(4,61)$ la califica como especie generalista. Esta especie tiene entonces una dieta semejante a T. laniger quien consume frutas, insectos y semillas (Lopez-Arevalo et al. 1993). Comparaciones con otras especies de Thomasomys son limitadas por el poco conocimiento de la dieta en este género (Pacheco en prensa).

La dieta herbívora de las dos especies de Thomasomys no está asociada a una especialización en dientes o estructura craneal semejante a la encontrada en roedores herbívoros de la puna como Andinomys, Chinchillula, Galenomys, Punomys, Neotomys y Phyllotis (Hershkovitz 1962), aunque varias especies de Thomasomys presentan molares con una moderada hipsodoncia (Voss 2003, Pacheco en prensa) que podría estar relacionado a la dieta herbívora.

La prevalencia de materia vegetal en estas especies de bosque montano concuerda con Suarez (1994) quien señala que la alta proporción de semillas y tejidos vegetales en el estómago de roedores estaría asociada a la fácil obtención de recursos en comparación a una dieta basada en proteínas.

Akodon orophilus es considerado una especie insectívora (90,1\% del volumen de la dieta) con preferencia hacia los artrópodos adultos $(\mathrm{CV}=19,9 \%)$, en su mayoría del orden Coleoptera. Se le califica también como especialista al obtener el valor más bajo con el Índice de Levins $(1,70)$, concordando con reportes previos que sugieren que el género Akodon es insectívoro. Así, A. boliviensis es reportado como una especie insectívora (Dorst 1972), al igual que $A$. subfuscus y $A$. torques (Solari 2007). Por otra parte, el mayor consumo de artrópodos larva durante la época húmeda en $A$. orophilus concuerda con estudios previos que indican que la dieta de roedores akodontinos usualmente presenta variación estacional (Glanz 1984, Meserve et al. 1988, Pizzimenti y De Salle 1980), lo cual está aparentemente relacionado con el presupuesto energético de las especies (Solari 2007).

Nuestro trabajo es al parecer el primero que reporta la dieta en
Microryzomys, encontrándose una clara tendencia a la omnivoría en $M$. altissimus y $M$. minutus; ambas especies consumieron una alta proporción de materia vegetal, $51,2 \%$ y $45,4 \%$ respectivamente; además del consumo de artrópodos adultos en $M$. altissimus $(35,92 \%)$ y $M$. minutus $(26,58 \%)$.

El análisis en relación al sexo y la época de colecta mostró que Akodon orophilus exhibe preferencias $(\mathrm{CV}<0,25)$ para el consumo de artrópodos adultos en cualquiera de las estaciones y que tanto machos como hembras prefieren el consumo de esta categoría; mientras que Thomasomys kalinowskii no mostró una preferencia alimenticia entre sexos.

La sobreposición de nicho entre pares de especies mostró valores menores a 0,75 en el $80 \%$ de los pares de especies, excepto entre $M$. altissimus - T. notatus $(0,822)$ y $M$. minutus - $T$. kalinowskii $(0,816)$. Valores altos de sobreposición de nicho indican una alta similaridad en la dieta de los pares de especies y una posible competencia entre ellas. La baja sobreposición de nichos encontrada generalmente entre las especies simpátricas de bosques montanos sugiere entonces una baja similaridad en la dieta y por ende una baja competencia en el uso de los recursos (Sale 1974). Nuestros resultados contrastan con un estudio similar realizado en el Altiplano del sur del Perú por Solari (2007), quien encuentra valores altos de sobreposición de nicho entre pares de especies de un ensamblaje de roedores con predominancia de akodontinos.

Se concluye que la dieta del ensamblaje de roedores sigmodontinos de los bosques montanos de Huánuco es diversa y heterogénea: $A$. orophilus es netamente insectívoro, $T$. notatus y T. kalinowskii son predominantemente herbívoros, mientras que $M$. altissimus y $M$. minutus muestran una tendencia a la omnivoría. Estas especies no evidencian una marcada competencia por los recursos alimenticios sino que tienden a utilizar la disponibilidad de recursos alimenticios de tal manera que les permite coexistir en el mismo tiempo y espacio. Aunque otros factores (v.g. tamaño del cuerpo, microhábitat, horario de actividad) pueden ser importantes para la segregación de nichos entre las especies (Meserve et al. 1988, Suárez \& Bonaventura 2001), nuestro estudio señala que el recurso alimenticio es un factor predominante, a la vez que no excluye que otros factores como microhábitats puedan ser también importantes. Falta de estudios similares nos impiden reconocer si estos resultados están relacionados al tipo de ambiente y la disponibilidad de recursos o a características propias de las especies. Los pocos estudios disponibles en los Andes peruanos difieren notablemente en composición de especies y limitan las comparaciones. Los trabajos de Pizzimenti y De Salle $(1980,1981)$ resaltaron la importancia de los hábitos omnívoros, aunque en su ensamblaje hubo predominancia de filotinos pequeños y medianos; mientras que los resultados de Solari (2007) fueron en un ensamblaje con predominancia de akodontinos; y nuestro trabajo en un ensamblaje taxonómicamente heterogéneo.

Este estudio, como otros sobre dieta, debe considerar que los componentes de la dieta no puede juzgarse sólo a partir de su abundancia en los contenidos estomacales, ya que algunos elementos poco frecuentes en la dieta pueden contribuir con elementos indispensables para la nutrición de los animales (Schmidt-Nielsen 1997). Por otra parte las proporciones obtenidas del contenido estomacal pueden no representar las condiciones frescas del volumen, según lo asumido a priori 
(Hansson 1970). Se sugiere continuar este tipo de estudios en otros bosques montanos para dilucidar si los resultados obtenidos son propios de este ambiente o de un ensamblaje particular de especies, y en otros ambientes y lugares para relacionar patrones de dieta con los distintos ensamblajes de roedores.

\section{Agradecimientos}

Parte de esta investigación fue presentada por M. NoblecillaHuiman para sustentar el título profesional de Biólogo en la Universidad Nacional Mayor de San Marcos, Lima, Perú; y fue parte del proyecto "Biodiversidad, Conservación, Impacto ambiental y Macrozonificación de los bosques montanos del Departamento de Huánuco" (Proyecto financiado por el Consejo Superior de Investigaciones de la UNMSM, otorgado a Víctor Pacheco). Agradecemos a Catherine Sahley, Lucía Luna, Sergio Solari, Elena Vivar y Richard Cadenillas por sus comentarios y sugerencias para este manuscrito. Igualmente, nuestro agradecimiento a Edith Arias y Malena Vílchez por su invaluable colaboración en la identificación de muestras vegetales y entomológicas respectivamente, y a María Peralta, Sandra Velazco, Carlos Tello, Jannina Milla, quienes obtuvieron las muestras biológicas. Finalmente, agradecemos el valioso apoyo de José D. Álvarez Huayta en la búsqueda de literatura, edición y otros aspectos que permitieron completar satisfactoriamente este trabajo.

\section{Literatura citada}

Brandán Z.J. 1995. Contribución al conocimiento de la dieta de Akodon simulator simulator (Thomas, 1916) (Rodentia: Cricetidae). Acta Zoológica Lilloana 43(1): 73-79.

Dorst J. 1972. Morphologie de l'estomac et regime alimentaire de quelques rongeurs des hautes Andes du Perou. Mammalia 36(4): 647-656.

Giannoni S.M., C. Borghi, M. Dacar \& C.M. Campos. 2005. Main food categories in diets of sigmodontine rodents in the monte (Argentina). Mastozoología Neotropical 12(2): 181-187.

Glanz W.E. 1984. Ecological relationships of two species of Akodon in central Chile. Journal of Mammalogy 65(3): 433-411.

Guabloche A., M. Arana \& O.E. Ramirez. 2002. Diet and gross gastric morphology of Oryzomys xantheolus (Sigmodontinae, Rodentia) in a Peruvian Loma. Mammalia 66(3): 405-411.

Hansson L. 1970. Methods of morphological diet micro-analysis in rodents. Oikos 21(2): 255-266.

Hershkovitz P. 1962. Evolution of Neotropical cricetine rodents (Muridae) with special reference to the phyllotine group. Fieldiana: Zoology 46: 1-525.

Horn H.S. 1966. Measurement of "overlap" in comparative ecological studies. American Naturalist 100(914): 419-424.

Levins R. 1968. Evolution in changing environments: Some theoretical explorations. Princeton University Press, Princeton, New Jersey, USA. 120 pp.

Lopez-Arevalo H., O. Montenegro-Díaz \& A. Cadena. 1993. Ecología de los pequeños mamíferos de la Reserva Biológica Carpanta, en la Cordillera Oriental Colombiana. Studies on Neotropical Fauna and Environment 28(4): 193-210.

López-Cortés F., A. Cortés, E. Miranda \& J. Rau. 2007. Dietas de Abrothrix andinus, Phyllotis xanthopygus (Rodentia) y Lepus europaeus (Lagomorpha) en un ambiente altoandino de Chile. Revista chilena de Historia Natural 80(1): 3-12.

Luna L. \& B.D. Patterson. 2003. A remarkable new mouse (Muridae: Sigmodontinae) from Southeastern Peru: with comments on the affinities of Rhagomys rufescens (Thomas, 1886). Fieldiana Zoology, new series 101: 1-24.

Meserve P.L. 1981. Trophic relationship among small mammals in a Chilean semiarid thorn scrub community. Journal of Mammalogy 62(2): 304-314.

Meserve P.L., B.K. Lang \& B.D. Patterson. 1988. Trophic relationships of small mammals in a Chilean temperate rainforest. Journal of Mammalogy 69(4): 721-730.

Pacheco V. 2002. Mamíferos del Perú. En: Diversidad y Conservación de los Mamíferos Neotropicales (G. Ceballos y J.A. Simonetti, Eds.), pp. 503-549. CONABIO-UNAM, México D.F., México.

Pacheco V. (en prensa). Genus Thomasomys Coues, 1884. In J.L. Patton, U.F.J. Pardiñas, and G. D'Elía, eds. Mammals of South America. Volume 2, Rodents. The University of Chicago Press, Chicago, IL.

Pacheco V. \& M. Peralta. 2011. Rediscovery of Rhipidomys ochrogaster J.A. Allen, 1901 (Cricetidae: Sigmodontinae) with a redescription of the species. Zootaxa 3106: 42-59.

Pacheco V., M. Peralta, C. Jiménez, S. Velazco, S. Mori, C. Tello, L. Huamaní, D. Vivas \& R. Ledesma. 2006. Biodiversidad, conservación, impacto ambiental y macrozonificación de los bosques montanos del departamento de Huánuco. Informe técnico final presentado a la Universidad Nacional Mayor de San Marcos.

Pacheco V., E. Salas, L. Cairampoma, M. Noblecilla, H. Quintana, F. Ortiz, P. Palermo y R. Ledesma. 2008 (2007). Diversidad y conservación de los mamíferos en la cuenca del río Apurímac, Perú. Revista Peruana de Biología 14(2): 169- 180.

Pacheco V., R. Cadenillas, E. Salas, C. Tello \& H. Zeballos. 2009. Diversidad y endemismo de los mamíferos del Perú. Revista Peruana de Biología 16(1): 005-032.

Pacheco V., G. Márquez, E. Salas \& O. Centty. 2011. Diversidad de mamíferos en la cuenca media del río Tambopata, Puno, Perú. Revista Peruana de Biología 18(2): 231-244.

Pianka E.R. 1973. The structure of lizard communities. Annual Review of Ecology and Systematics 4: 53-74.

Pizzimenti J.J. \& R. De Salle. 1980. Dietary and morphometric variation in some Peruvian rodent communities: the effect of feeding strategy on evolution. Biological Journal of the Linnean Society 13(4): 263-285.

Pizzimenti J.J. \& R. De Salle. 1981. Factors influencing the distributional abundance of two trophic guilds of Peruvian cricetid rodents. Biological Journal of the Linnean Society 15(4): 339-354.

Sale P. F. 1974. Overlap in resource use, and interspecific competition. Oecologia 17(3): 245-256.

Schmidt-Nielsen K. 1997. Animal physiology: adaptation and environment. 5th ed. Cambridge University Press, Cambridge. $607 \mathrm{pp}$.

Solari S. 1997. Relaciones tróficas en una comunidad de roedores altoandinos en el Parque Nacional Manu. Tesis para optar el Título Profesional de Biólogo con mención en Zoología. UNMSM.

Solari S. 2007. Trophic relationships within a Highland rodent assemblage from Manu National Park, Cusco, Peru. In D. A. Kelt, E. P. Lessa, J. Salazar-Bravo, and J. L. Patton, eds. The Quintessential Naturalist: Honoring the Life and Legacy of Oliver P. Pearson. University of California Publications in Zoology 134: 225-240.

Suarez O.V. 1994. Diet and habitat selection of Oxymycterus rutilans (Rodentia, Cricetidae). Mammalia 58(2): 225-234.

Suárez, O.V. \& S.M. Bonaventura. 2001. Habitat use and diet in sympatric species of rodents of the low Parana delta, Argentina. Mammalia 65: 167-176.

Voss R.S. 2003. A new species of Thomasomys (Rodentia: Muridae) from eastern Ecuador, with remarks on mammalian diversity and biogeography in the Cordillera Oriental. American Museum Novitates 3421: 1-47.

Vorontsov N.N. 1961. Variation in the transformation rates of or- 
gans of the digestive systems in rodents and the principle of functional compensation. Evolutionary Morphology: 49-52 (translated from Doklady Akad. Nauk SSRR 136:1494-1497).

Young K. R. \& N. Valencia. 1992. Introducción: Los Bosques
Montanos del Perú. In K.R. Young y N. Valencia, eds. Biogeografía, Ecología y Conservación del Bosque Montano en el Perú. Memorias del Museo de Historia Natural, UNMSM 21: 5-9.

Anexo 1. Ocurrencia de plantas y artrópodos en la dieta de los roedores sigmodontinos de los bosques montanos de Huánuco, Perú.

\begin{tabular}{|c|c|c|c|c|c|}
\hline \multirow{2}{*}{ Categoría alimenticia } & A. orophilus & M. altissimus & M. minutus & T. notatus & T. kalinowskii \\
\hline & $(\mathrm{N}=37)$ & $(\mathrm{N}=5)$ & $(\mathrm{N}=9)$ & $(\mathrm{N}=6)$ & $(\mathrm{N}=48)$ \\
\hline \multicolumn{6}{|l|}{ Monocotyledoneae } \\
\hline \multicolumn{6}{|l|}{ Poaceae } \\
\hline Glumas-lemnas, raíz, semilla & & & & & $X$ \\
\hline Hoja-epidermis, tallo, fruto, espigilla & $x$ & $\mathrm{X}$ & $x$ & $X$ & $X$ \\
\hline \multicolumn{6}{|l|}{ Cyperaceae } \\
\hline Hoja-epidermis, tallo, fruto & $X$ & & $X$ & $X$ & $X$ \\
\hline \multicolumn{6}{|l|}{ Dicotiledoneas } \\
\hline \multicolumn{6}{|l|}{ Asteraceae } \\
\hline Hoja-epidermis, aquenios, semilla, fruto & & & & & $x$ \\
\hline \multicolumn{6}{|l|}{ Piperaceae } \\
\hline Semillas enteras & & & & & $X$ \\
\hline $\begin{array}{l}\text { Hoja-epidermis, semilla restos, tallo, raíz, } \\
\text { estambres, pericarpo de fruto }\end{array}$ & & & & & $X$ \\
\hline \multicolumn{6}{|l|}{ Insecta } \\
\hline Coleoptera & & & $X$ & & $X$ \\
\hline Carabidae & $X$ & $X$ & & $X$ & \\
\hline Coccinellidae & $X$ & & & & \\
\hline Curculionidae & $x$ & & & & \\
\hline Scarabaeidae & $X$ & & & & \\
\hline \multicolumn{6}{|l|}{ Hemiptera } \\
\hline Pentatomidae & $X$ & & & & \\
\hline Cicadelidae & $x$ & & & & \\
\hline \multicolumn{6}{|l|}{ Lepidoptera } \\
\hline Nymphalidae & $X$ & & & & \\
\hline \multicolumn{6}{|l|}{ Diptera } \\
\hline Cecidomyiidae & $X$ & & & & \\
\hline Hymenoptera & $X$ & & & & \\
\hline Phthiraptera & $X$ & & & & \\
\hline Diplura & $x$ & & & & \\
\hline Arachnida & $X$ & & & & \\
\hline
\end{tabular}

